\title{
Impact of boron and indium doping on the structural, electronic and optical properties of $\mathrm{SnO}_{2}$
}

\author{
Petros-Panagis Filippatos ${ }^{1,2}$, Nikolaos Kelaidis ${ }^{1,2}$, Maria Vasilopoulou ${ }^{1}$, \\ Dimitris Davazoglou ${ }^{1} \&$ Alexander Chroneos ${ }^{2,3 凶}$
}

Tin dioxide $\left(\mathrm{SnO}_{2}\right)$, due to its non-toxicity, high stability and electron transport capability represents one of the most utilized metal oxides for many optoelectronic devices such as photocatalytic devices, photovoltaics (PVs) and light-emitting diodes (LEDs). Nevertheless, its wide bandgap reduces its charge carrier mobility and its photocatalytic activity. Doping with various elements is an efficient and low-cost way to decrease $\mathrm{SnO}_{2}$ band gap and maximize the potential for photocatalytic applications. Here, we apply density functional theory (DFT) calculations to examine the effect of p-type doping of $\mathrm{SnO}_{2}$ with boron (B) and indium (In) on its electronic and optical properties. DFT calculations predict the creation of available energy states near the conduction band, when the dopant (B or In) is in interstitial position. In the case of substitutional doping, a significant decrease of the band gap is calculated. We also investigate the effect of doping on the surface sites of $\mathrm{SnO}_{2}$. We find that $\mathrm{B}$ incorporation in the (110) does not alter the gap while In causes a considerable decrease. The present work highlights the significance of $\mathrm{B}$ and In doping in $\mathrm{SnO}_{2}$ both for solar cells and photocatalytic applications.

Tetragonal $\mathrm{SnO}_{2}$ is a wide bandgap semiconductor, which typically exhibits n-type conductivity due to the oxygen vacancies that are created during the crystallization process ${ }^{1-4}$. $\mathrm{SnO}_{2}$ is commonly used for glazes ${ }^{1}$, polishing powder ${ }^{2}$, photovoltaics ${ }^{3}$, and gas sensors ${ }^{4}$. Recently, $\mathrm{SnO}_{2}$ has been demonstrated as a cathode material in Li-ion batteries ${ }^{5}$. Wang et al. ${ }^{5}$ have shown that $\mathrm{Sn}_{2} \mathrm{O}_{3}$ in $\mathrm{SnO}_{2}$ nanosheets significantly improves battery performance. However, the common $\mathrm{SnO}_{2}$ films exhibit low intrinsic carrier mobility, which has been attributed to deep donor states originating from oxygen vacancies ${ }^{3}$. Many doping strategies have been adopted to further increase its electrical conductivity and improve its absorption in the visible region-for photocatalytic applications-by reducing its wide energy gap. Specifically, many experimental and theoretical works have demonstrated that halogen doping in $\mathrm{SnO}_{2}$ increases conductivity and transparency, making it a better candidate for optoelectronic applications ${ }^{6,7}$.

Another option for improving the properties the properties of $\mathrm{SnO}_{2}$ is doping with p-type elements ${ }^{8}$. Transparent conducting oxides (TCOs) fabricated from doped semiconductor oxides such us In:SnO $\mathrm{S}_{2}(\mathrm{ITO}), \mathrm{F}: \mathrm{SnO}_{2}$ (FTO) and $\mathrm{B}: \mathrm{ZnO}$ (BZO) are commonly used as transparent conductive materials in industrial applications such as displays and lighting devices ${ }^{8}$. In these structures, the metal atom is typically substituted by the dopant, which improves the charge carrier conductivity. Experimental reports on mesoporous oxides indicate remarkable chemical properties when B occupies an interstitial position ${ }^{9}$. These B interstitials $\left(B_{i}\right)$ enhance the carrier densities of the semiconductors and lead to a diverse coordination environment with hyperstoichiometric oxygen vacancies. Additionally, some reports propose $\mathrm{B}$ as a doping candidate for $\mathrm{SnO}_{2}$ based photocatalytic applications ${ }^{10}$. In particular, B atoms can occupy interstitial positions creating a hybridization of the B-p orbital with the nearest O-2p orbital ${ }^{11}$. This improves the activity of catalysts as it reduces the $\mathrm{e}^{-} / \mathrm{h}^{+}$recombination $^{12}$. Tran et al. ${ }^{13}$ studied the optical and the electrical properties of $\mathrm{B}: \mathrm{SnO}_{2}$ revealing that the transmittance of the films is increased with the increase of the B dopant concentration. This translates into a decrease of the band gap as compared to undoped $\mathrm{SnO}_{2}$, which make the material applicable for photocatalytic devices. Interestingly, they reported the dependance of the transmittance with the crystallization temperature, which can be attributed to the increased scattering of photons due to the larger grains as well as the increase of tin and oxygen vacancies.

${ }^{1}$ Institute of Nanoscience and Nanotechnology (INN), National Center for Scientific Research Demokritos, 15310 Agia Paraskevi, Athens, Greece. 2Faculty of Engineering, Environment and Computing, Coventry University, Priory Street, Coventry CV1 5FB, UK. ${ }^{3}$ Department of Materials, Imperial College, London SW7 2AZ, UK. ${ }^{\square}$ email: alexander.chroneos@imperial.ac.uk 
Regarding the electrical properties of the material, it was determined that $\mathrm{B}$ doping beneficially increases the carrier concentration compared to the undoped $\mathrm{SnO}_{2}{ }^{13}$.

Indium is an important dopant used to increase the electrical and optical properties of $\mathrm{SnO}_{2}{ }^{14-16}$. Aouaj et al. ${ }^{14}$ compared the electrical and optical properties of ITO and FTO and they determined that at high In content the absorbance is lower in ITO and the gap value is increased. Additionally, they predicted that that the electrical resistivity is generally lower in ITO as compared to FTO. Finally, they concluded that ITO at different In concentration can be used as a transport layer or electrode in solar cells. Similar experiments of Kulkarni et al. ${ }^{15}$ showed that the deposition temperature and substrate play a major role regarding the optical properties of ITO and variation to the refractive index value.

Apart from the experimental works, there are also theoretical studies based on DFT that examine the influence of doping in $\mathrm{SnO}_{2}$, mostly in substitutional positions ${ }^{16-24}$. Especially for the halogen substitutional doping, it is revealed that single donor states arise inside the bandgap; however, the effect of interstitials is not generally taken into consideration by the community. Mallick et al. ${ }^{23}$ examined theoretically the impact of substitutional $\mathrm{Al}$ in $\mathrm{SnO}_{2}$. They showed that the introduction of aluminium (Al) leads to new defect states above the Fermi energy level which are highly depend on the doping concentration. The band gap of $\mathrm{Al}: \mathrm{SnO}_{2}$ remains unchanged for low doping concentrations (below 1.85\%) while there is a small increase in high concentrations (higher than 3.70\%). Duan et al. ${ }^{24}$ investigated the effect of gallium $(\mathrm{Ga})$ in $\mathrm{SnO}_{2}$ and determined that Ga substitutionals in $\mathrm{Sn}$ sites, shifts the Fermi energy to the valence band and introduces more charge holes at the Ga sites. The Ga doping also slightly reduces the band gap and increases the electrical conductivity of the material. Nevertheless, the effect of $\mathrm{B}$ and In incorporation in $\mathrm{SnO}_{2}$ is not extensively examined using computational modelling techniques. From all the above it is concluded that the B family group elements (group IIIA of the periodic table) introduce energy states in the band gap which may play a major role in photovoltaic and photocatalytic devices.

In most previous DFT studies, the bandgap of $\mathrm{SnO}_{2}$ is considerably underestimated and predicted at a value of $1-2 \mathrm{eV}^{22,25}$. This is a well-known deficiency of the Local Density Approximation (LDA) and of the Generalized Gradient Approximation (GGA). Therefore, the use of computationally demanding but more accurate hybrid exchange-correlation functionals are deemed necessary to accurately describe the band gap and the position of states in the gap or at its edges.

In the present study, we apply Hybrid functional DFT calculations using $\mathrm{PBE}^{45}$ to attain a bandgap value of $3.35 \mathrm{eV}$, which agrees well with the experimentally reported bandgap ${ }^{3,26}$. More analytically, we examine the effect of $\mathrm{B}$ and In doping on the bulk $\mathrm{SnO}_{2}$ and we also investigate the effect of interstitial doping of the (110) surface for the first time. Density of states (DOS) calculations showed a reduction of the band gap in all the substitutional cases and the formation of energy states in the bandgap for the substitutional and interstitial doping. This bandgap reduction combined with the created inter-gap energy levels are highly beneficial for the photocatalytic applications of $\mathrm{SnO}_{2}$ while the predicted characteristics can also be applied in photovoltaic technologies.

\section{Results}

Bulk rutile $\mathrm{SnO}_{2}$. $\quad \mathrm{SnO}_{2}$ forms in the $\mathrm{P}_{2} / \mathrm{mmm}$ space group crystalizing in the rutile structure. The experimental unit cell parameters $\mathrm{a}=\mathrm{b}=4.737 \AA$ and $\mathrm{c}=3.186 \AA$ were determined using X-ray diffraction (XRD) experiments ${ }^{16}$. The calculated lattice parameters after the relaxation of rutile $\mathrm{SnO}_{2}$ are $\mathrm{a}=\mathrm{b}=4.717 \AA$ and $\mathrm{c}=3.189 \AA$ in very good agreement with the experimental values. The dopant percentage of the present calculations is $1 \mathrm{~B}$ or In atom in $48 \mathrm{SnO}_{2}$ atoms, which results in $2.08 \%$ doping. Zhang et al. ${ }^{27}$ performed experiments regarding the effect of $\mathrm{B}$ in tin oxide and they predicted that $\mathrm{B}$ can either be at a $\mathrm{Sn}$ substitutional site or occupy an interstitial site. Zhang et al. ${ }^{27}$ determined that with this amount of doping the thickness of the film is reduced in parallel with the mean crystal size. We predict that in its Sn substitutional site the B atom is at a distance of $1.775 \AA$ from the nearest oxygen atom (refer to Fig. 1a) while for the interstitial case, the boron atom is a distance of $1.475 \AA$ from the O-atom (refer to Fig. 1b). In Fig. 1c,d we show the $\mathrm{In}_{\mathrm{Sn}}: \mathrm{SnO}_{2}$ and $\operatorname{In}_{\mathrm{i}}: \mathrm{SnO}_{2}$ cases, respectively. The substitutional dopant is relaxed at a distance of $2.11 \AA$ from the nearest oxygen atom, while the interstitial is located at a distance of $2.03 \AA$. In Fig. 1e we present the bulk supercell used. In Table 1, we report the relevant lattice parameters and volume changes, through doping, for the bulk system. Concerning the $\mathrm{B}: \mathrm{SnO}_{2}$ cases, there is a small decrease in the lattice volume for the substitutional case, which can be attributed to the smaller ionic radius of $\mathrm{B}$ than $\mathrm{Sn}\left(\mathrm{B}^{3+}: 0.23 \AA, \mathrm{Sn}^{4+}: 0.71 \AA\right)$ while for the interstitial case, there is an increase. The present results agree with the available experimental data that predict a decrease of the lattice parameters for the $\mathrm{B}_{\mathrm{Sn}}: \mathrm{SnO}_{2}$ and an increase for the $\mathrm{B}_{\mathrm{i}}: \mathrm{SnO}_{2}{ }^{9}$. Similarly for $\mathrm{In}: \mathrm{SnO}_{2}$ we predicted that in all the cases, there is an increase to the lattice parameters. This is in agreement with previous experimental studies ${ }^{28}$.

For each doping case, we calculate the DOS and the PDOS and present them in Fig. 2. The DOS of the undoped $\mathrm{SnO}_{2}$ is given in Fig. 2e for reference. To have a clear and more reliable picture of the changes of the electronic structure due to doping, we employed the hybrid functional PBE0, which provides a good bandgap value (calculated at $3.35 \mathrm{eV}$ ) and is in agreement with the experimental value ${ }^{26}$.

In Fig. $2 \mathrm{a}$, the $\mathrm{B}_{\mathrm{Sn}}$ :SnO $\mathrm{Sn}_{2}$ case is examined. In this case, it is observed from the total DOS (grey) that the bandgap is reduced to the value of $2.73 \mathrm{eV}$ while some energy states are created inside the bandgap at $1 \mathrm{eV}$. Furthermore, it is seen that some additional states arise at the valence band maximum. Looking at the PDOS of the B substitutional case, we predict that these states are created mostly from the hybridization of O-2p with Sn-5p and the B-2p orbitals. This is in agreement with the work of Yu et al. ${ }^{29}$, which also predicts mid-gap states and gap reduction when $\mathrm{B}$ is inserted. However, in their study, there is an underestimation to the bandgap value of $\mathrm{SnO}_{2}$. Experimental studies ${ }^{30}$ have shown that $2 \% \mathrm{~B}$ doping decreases the bandgap, which is in good agreement with the present study.

To further investigate the effect of $\mathrm{B}$ doping, we considered interstitial $\mathrm{B}$ in $\mathrm{SnO}_{2}$. We expect that the interstitial incorporation of $\mathrm{B}$ results in gap states forming to the conduction band edge between 1.66 and $3.49 \mathrm{eV}$. 


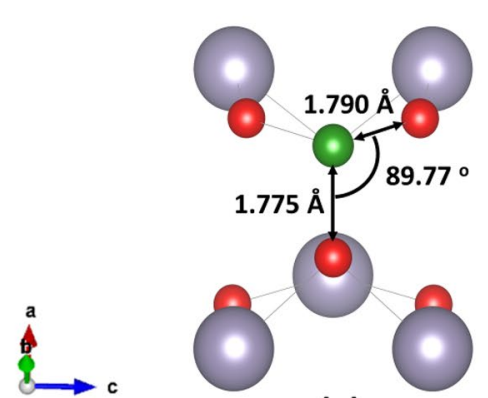

(a)
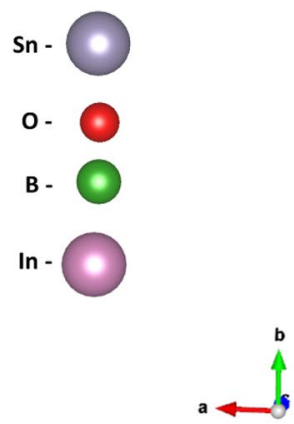

(b)
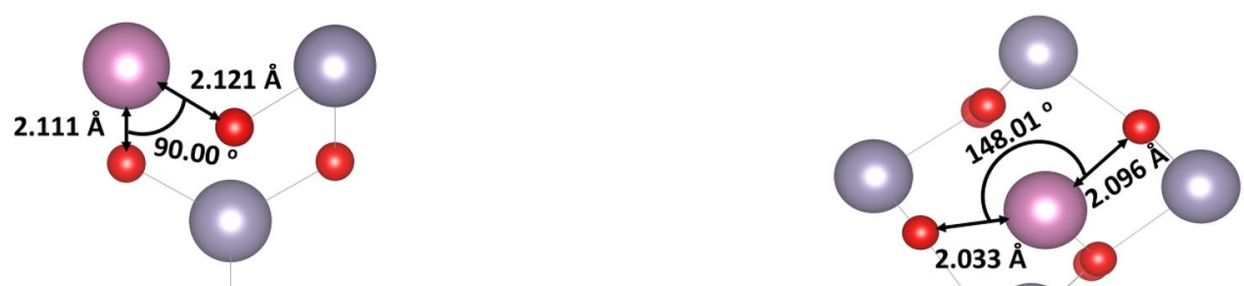

$\stackrel{a}{c}$

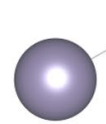

○

(c)
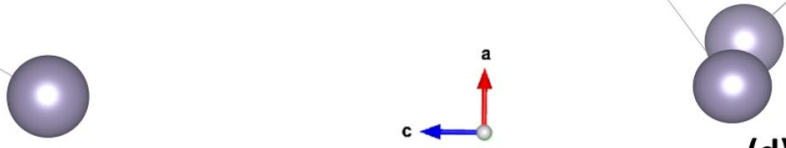

(d)

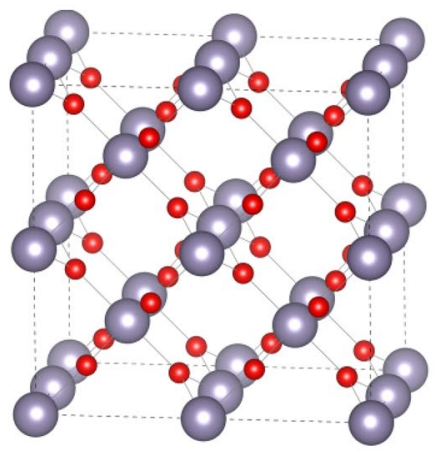

(e)

Figure 1. The structures of (a) boron substitutional doped $\mathrm{SnO}_{2}\left(\mathrm{~B}_{\mathrm{Sn}_{n}}: \mathrm{SnO}_{2}\right),(\mathbf{b})$ boron interstitial doped $\mathrm{SnO}_{2}$ $\left(\mathrm{B}_{\mathrm{i}}: \mathrm{SnO}_{2}\right),(\mathbf{c})$ indium substitutional doped $\mathrm{SnO}_{2}\left(\mathrm{In}_{\mathrm{Sn}}: \mathrm{SnO}_{2}\right)$, (d) indium interstitial doped $\mathrm{SnO}_{2}\left(\mathrm{In}_{\mathrm{i}}: \mathrm{SnO}_{2}\right)$ and (e) the bulk supercell.

\begin{tabular}{|l|l|l|l|}
\hline & a $(\AA)$ & c $(\AA)$ & Vol $\left(\AA^{3}\right)$ \\
\hline $\mathrm{SnO}_{2}$ & 4.717 & 3.189 & 70.956 \\
\hline $\mathrm{B}_{\mathrm{Sn}}: \mathrm{SnO}_{2}$ & 4.674 & 3.153 & 68.881 \\
\hline $\mathrm{B}_{\mathrm{i}}: \mathrm{SnO}_{2}$ & 4.866 & 3.225 & 76.361 \\
\hline $\mathrm{In}_{\mathrm{Sn}}: \mathrm{SnO}_{2}$ & 4.730 & 3.194 & 71.459 \\
\hline $\mathrm{In}_{\mathrm{i}}: \mathrm{SnO}_{2}$ & 4.901 & 3.220 & 77.343 \\
\hline
\end{tabular}

Table 1. The calculated lattice constants and cell volumes for all the dopants. 

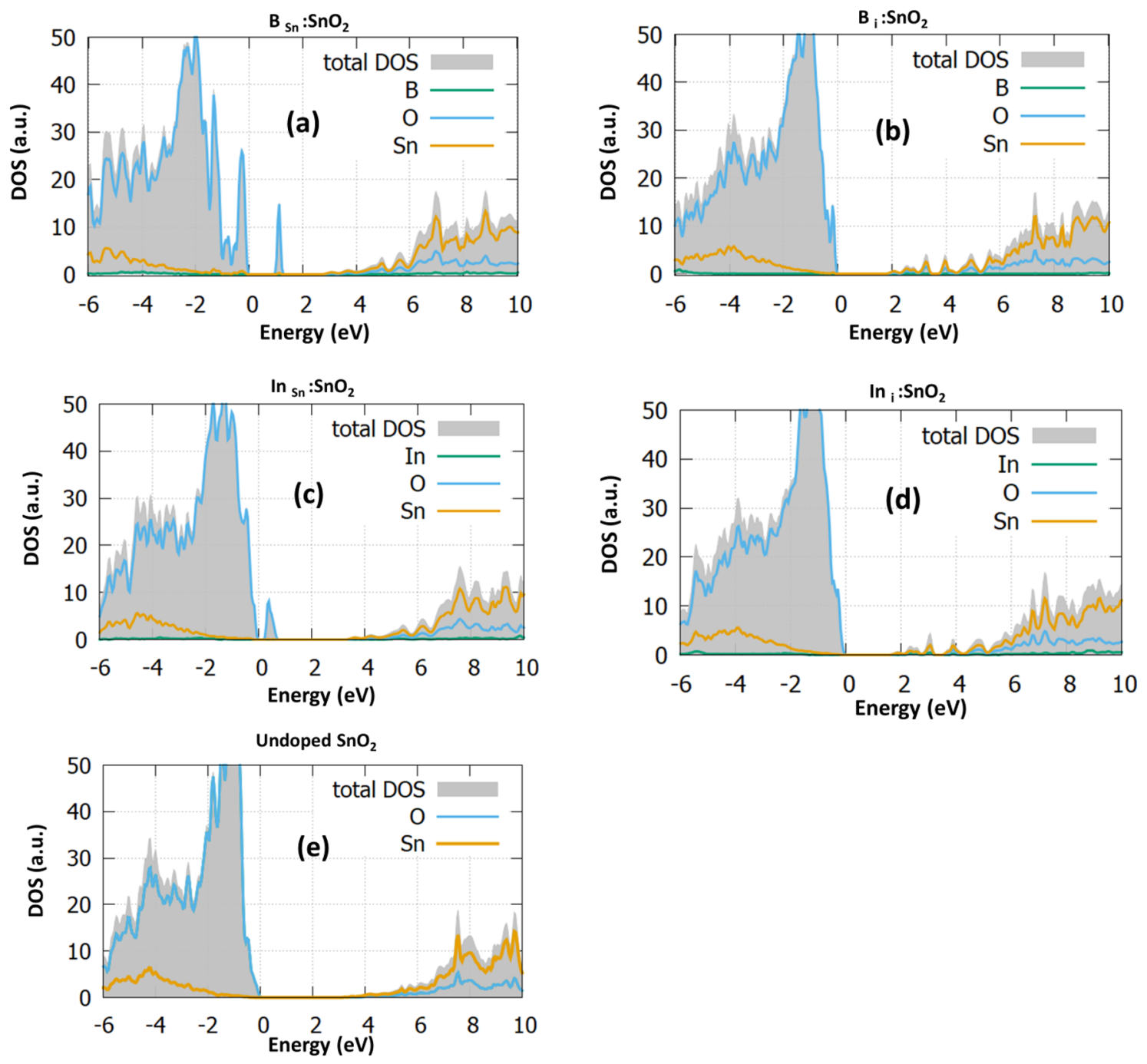

Figure 2. The total density of states (DOS) and the projected density of states (PDOS) of (a) $\mathrm{B}_{\mathrm{Sn}_{\mathrm{n}}}: \mathrm{SnO}_{2},(\mathbf{b})$ $\mathrm{B}_{\mathrm{i}}: \mathrm{SnO}_{2},(\mathbf{c}) \mathrm{In}_{\mathrm{Sn}}: \mathrm{SnO}_{2},(\mathbf{d}) \mathrm{In}_{\mathrm{i}}: \mathrm{SnO}_{2}$ and (e) undoped $\mathrm{SnO}_{2}$.

The bandgap is seen to increase to the value of $3.72 \mathrm{eV}$. As it is discussed in previous studies, the formation of energy states in the middle of the bandgap is highly beneficial for photocatalytic applications, but still, they can be a crucial disadvantage for PV and LED devices as they work as "traps" which reduce the device photocurrent and the photogenerated charge carriers ${ }^{30,31}$. Although $\mathrm{B}_{\mathrm{i}}: \mathrm{SnO}_{2}$ has not been examined theoretically, Zhi et al. ${ }^{9}$ predicted that interstitial atomic positions play a significant role in increasing the capacity of tin oxide, making it a good fit for supercapacitor applications. Zhi et al. ${ }^{9}$ used $4 \% \mathrm{~B}$ and they concluded that the interstitial atoms slightly increased the bandgap. This is the Moss-Burstein effect and it is generally seen in heavily doped semiconductors, where after a doping concentration, the bandgap of the semiconductor starts to increase and can even reach higher values than the undoped material ${ }^{32}$. In the case of $\mathrm{B}_{\mathrm{i}}: \mathrm{SnO}_{2}$, this provides a sufficient number of charge carriers that improve the capacitance of $\mathrm{SnO}_{2}{ }^{33}$. We predicted a bandgap increase in the case of the bulk $\mathrm{SnO}_{2}$ while in the (110) surface of $\mathrm{SnO}_{2}$ the gap remained unchanged. The (110) plane is considered the most highly energetic plane of the experimental $\mathrm{SnO}_{2}$ structure ${ }^{9,34,35}$ as we discuss in the next section. In essence the present calculations complete the DFT work of Zhi et al. ${ }^{9}$, which focused only to the (002) plane. Zhang et al. ${ }^{27}$ also performed electrical measurements to predict the properties of $\mathrm{B}: \mathrm{SnO}_{2}$. They predicted that in the interstitial position, $\mathrm{B}$ releases three free electrons resulting in an increase of the free electron concentration. Tran et al. ${ }^{13}$ predicted that the bandgap of $\mathrm{SnO}_{2}$ is decreased with the $\mathrm{B}$ incorporation and with the increase of the temperature. While this is opposite to what we predicted or other experimental works ${ }^{9}$ we believe that this is due to the effect of intrinsic defects, such as oxygen vacancies, which affect the gap value. Also, the percentage of $\mathrm{B}$ atoms that reside in substitutional sites play an important role to the increase of the transmission of light within $\mathrm{SnO}_{2}$. However, it is clear that even in this case the Moss-Burstein effect is still seen as the band gap is increasing after the 4 at.\% doping percentage.

Continuing with the bulk $\mathrm{In}: \mathrm{SnO}_{2}$ we examined the interstitial and substitutional formation. Focusing on the DOS of $\mathrm{In}_{\mathrm{Sn}}: \mathrm{SnO}_{2}$ (Fig. 2c) we predicted again unoccupied energy states near the valence band at $0.8 \mathrm{eV}$, which are created due to the hybridization of O-2p with Sn-5p and In-5p. These gap states might be beneficial 

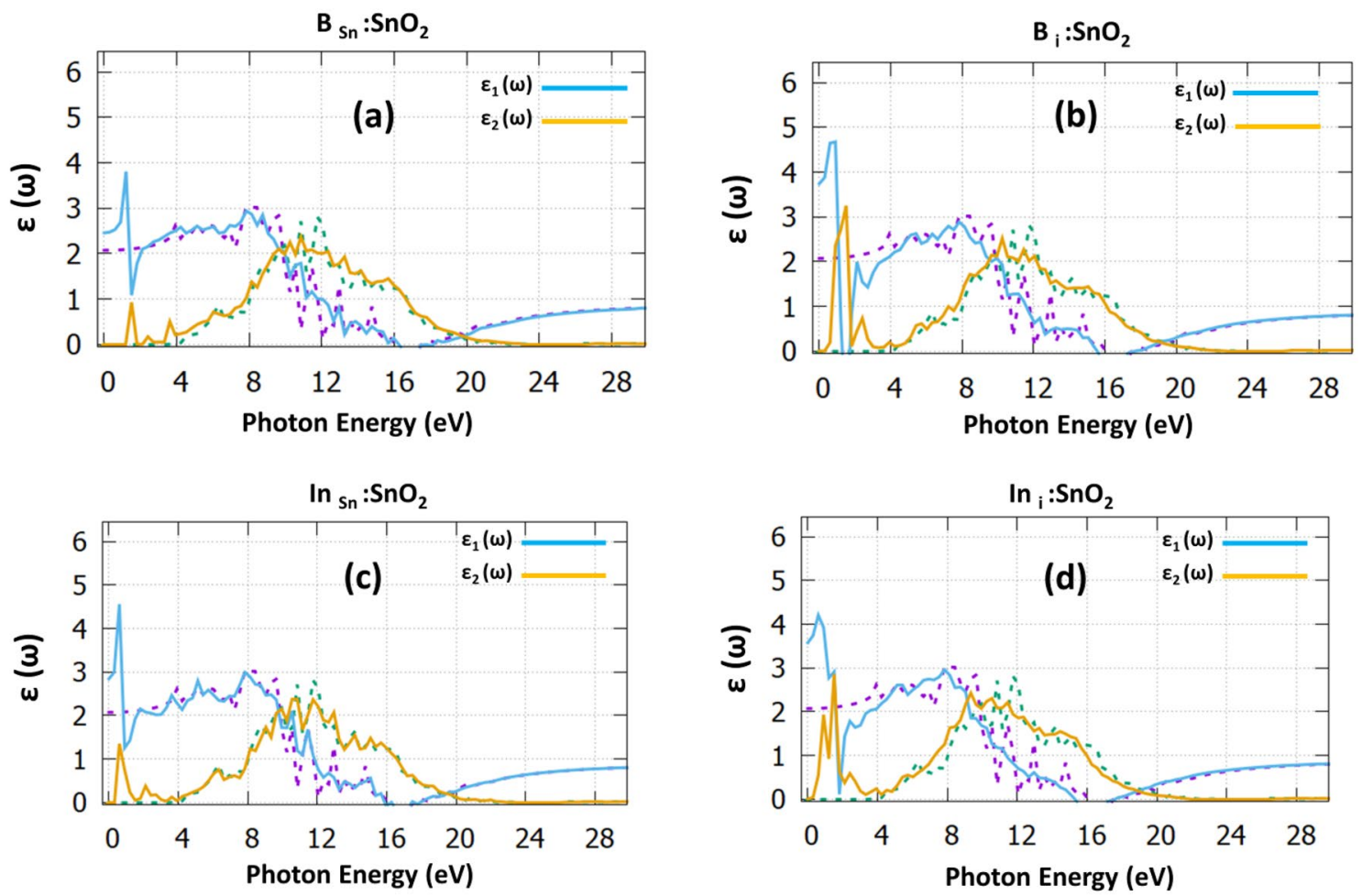

Figure 3. The dielectric function for (a) $\mathrm{B}_{\mathrm{Sn}}: \mathrm{SnO}_{2},(\mathbf{b}) \mathrm{B}_{\mathrm{i}}: \mathrm{SnO}_{2}$, (c) $\mathrm{In}_{\mathrm{Sn}}: \mathrm{SnO}_{2}$ and $(\mathbf{d}) \mathrm{In}_{\mathrm{i}}: \mathrm{SnO}_{2}$. The dotted purple and dotted green, correspond to the dielectric function of the undoped $\mathrm{SnO}_{2}$.

for device fabrication as they will serve as a route for the transition from the valence to the conduction band. Furthermore, now the bandgap is slightly decreased at a value of $3.25 \mathrm{eV}$. Thereafter, we performed calculations for the interstitial doping of indium in $\mathrm{SnO}_{2}$. We predict that the In-5p orbitals play a significant role in shifting the conduction band and reducing the band gap, which reaches a value of $3.48 \mathrm{eV}$. Similarly to the $\mathrm{B}$ case, the interstitial doping gives rise to energy levels inside the gap in the area between 1.48 and $3.29 \mathrm{eV}$. The present results agree well with the experimental data of Abdulsattar et al. ${ }^{36}$, which predicted a slight decrease to the bandgap with a doping percentage of $2 \%$. All these results show that although $\mathrm{B}: \mathrm{SnO}_{2}$ shows energy states that are detrimental for device applications, it is evident that gap reduction makes it possible for photocatalytic applications. In contrast, $\mathrm{In}: \mathrm{SnO}_{2}$ shows characteristics that make it a good candidate for an electron transport layer material for photovoltaic devices.

To consider possible applications of the doped bulk structures, we calculated their optical properties. In order to describe the absorption and dispersion mechanisms from the occupied to the empty electron levels, we predicted the dielectric function, presented in Fig. 3. Focusing on the real part of the dielectric function for the undoped $\mathrm{SnO}_{2}$ (dotted purple), it is seen that it reaches a maximum value of $8.5 \mathrm{eV}$. The negative value at $16.5 \mathrm{eV}$ is attributed to the metallic properties of undoped $\mathrm{SnO}_{2}{ }^{37,38}$. The imaginary part of the dielectric function provides helpful information regarding the probability of photon absorption. We calculated that there is a decreasing trend for all the cases due to Maxwell-Wagner interfacial polarization ${ }^{39}$. From the undoped $\mathrm{SnO}_{2}$ case (dotted green), two sharp peaks arise due to the transitions from $\mathrm{O}-2 p$ to $\mathrm{Sn}-5 p$. The dielectric constant of the undoped case is predicted at 2 in good agreement with previous experimental ${ }^{37}$ and theoretical works ${ }^{38}$. Focusing on $\mathrm{B}_{\mathrm{Sn}}: \mathrm{SnO}_{2}$ (refer to Fig. 3a) case, we predicted the dielectric constant is significantly increased to the value of 2.5. The major peak that arises at $1.7 \mathrm{eV}$ of the imaginary part can be attributed to the transition of electrons of B- $2 p$ and Sn-5p. Continuing with the interstitial B case (refer to Fig. 3b), there is a considerable increase to the dielectric constant, which reaches a value of 3.8 .

Regarding the $\mathrm{In}_{\mathrm{Sn}}: \mathrm{SnO}_{2}$ (Fig. 3c) the dielectric constant is increased to the value of 3, which is significantly higher than the $\mathrm{B}_{\mathrm{Sn}}: \mathrm{SnO}_{2}$ and finally, for the $\mathrm{In}_{\mathrm{i}}: \mathrm{SnO}_{2}$ (Fig. 3d) the dielectric constant reaches the value of 3.7. From Fig. 3 it can also be observed that there is a shift of the low photon energy peaks towards the visible region accompanied by an increase of the value of the dielectric constant. The recombination of charge carriers decreases, where the static dielectric constant monitors the electric fields inside the material by polarization. As a result, both $\mathrm{B}_{\mathrm{i}}: \mathrm{SnO}_{2}$ and $\mathrm{In}_{\mathrm{i}}: \mathrm{SnO}_{2}$ are highly preferred to use as heterojunction buffer layers in photovoltaic applications.

The refractive index is shown in Fig. 4. For the $\mathrm{SnO}_{2}$ the refractive index is computed in zero frequency at the value of 1.40 , which agrees with previous simulation studies ${ }^{38}$, but it is underestimated compared to the experimental value of $1.70^{40}$.

Regarding the doping structures, we predicted a value of 1.55 for $\mathrm{B}_{\mathrm{Sn}}: \mathrm{SnO}_{2}$ (Fig. 4a), while for $\mathrm{B}_{\mathrm{i}}: \mathrm{SnO}_{2}$ (Fig. 4b), there is an increase reaching a value of 1.95. Considering In doping, we predicted a value of 1.6 for the substitutional case (Fig. 4c) and a value of 1.85 for the interstitial In (Fig. 4d). It is evident that for the refractive 

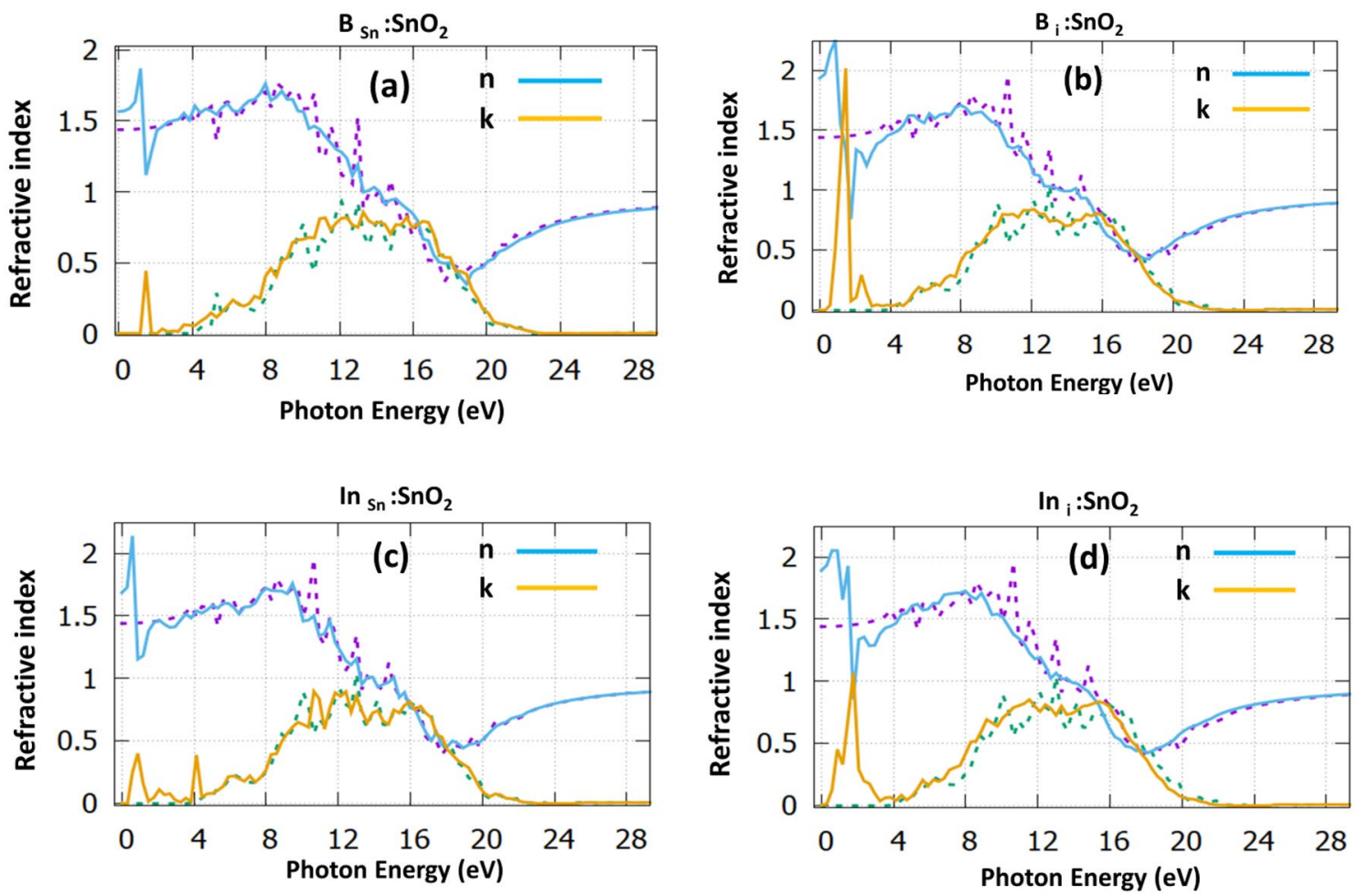

Figure 4. The refractive index for (a) $\mathrm{B}_{\mathrm{Sn}}: \mathrm{SnO}_{2}$, (b) $\mathrm{B}_{\mathrm{i}}: \mathrm{SnO}_{2}$, (c) $\mathrm{In}_{\mathrm{Sn}}: \mathrm{SnO}_{2}$ and (d) $\mathrm{In}_{\mathrm{i}}: \mathrm{SnO}_{2}$. The dotted purple and dotted green, correspond to the dielectric function of the undoped $\mathrm{SnO}_{2}$.
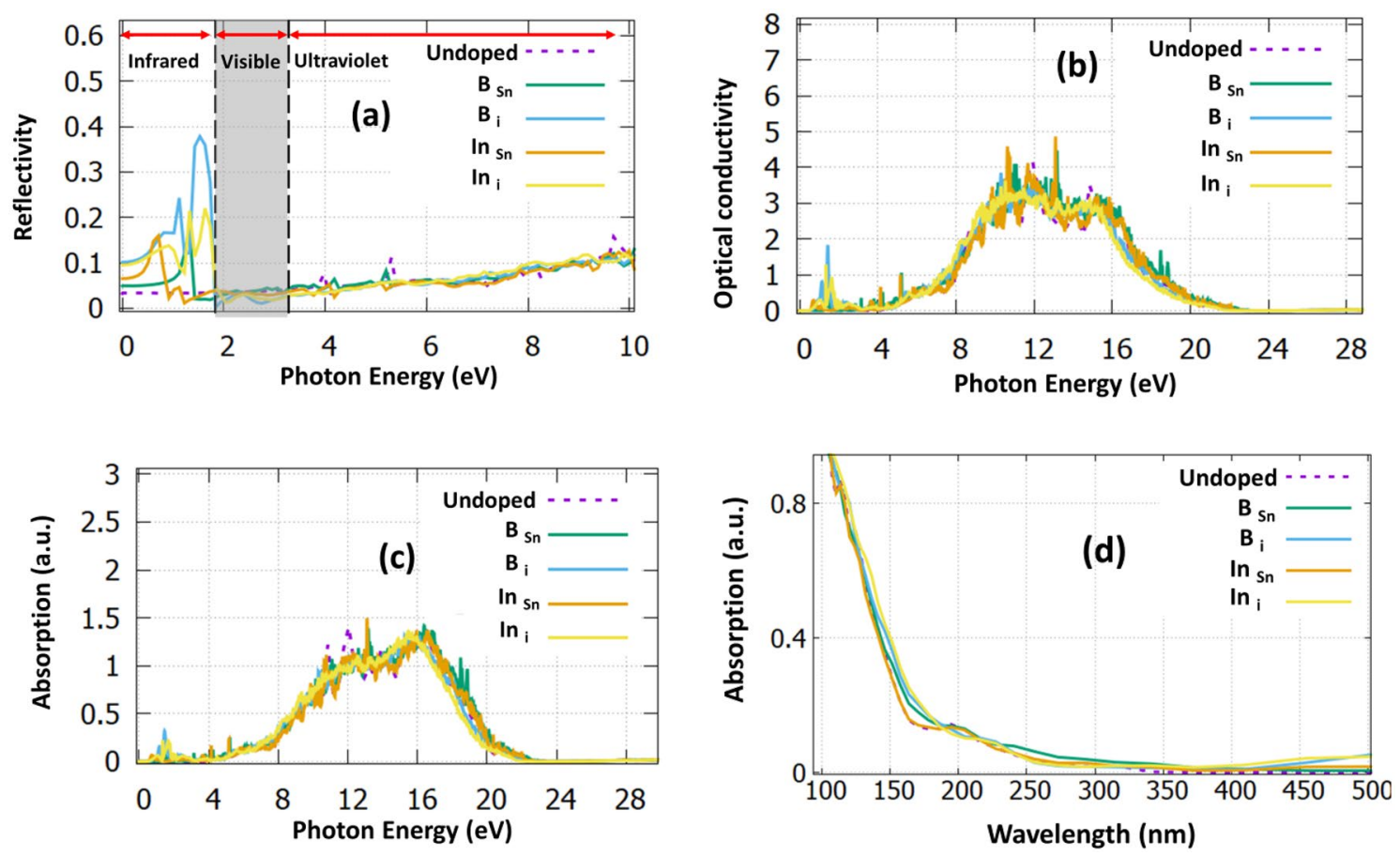

Figure 5. (a) The reflectivity for all the doped structures (b) The optical conductivity for all the studied structures (c) the absorption coefficient for all the structures versus the photon energy (d) the absorption coefficient for all the structures versus the wavelength. The dotted purple and dotted green, correspond to the undoped $\mathrm{SnO}_{2}$. 


\begin{tabular}{|l|l|l|l|l|}
\hline & Bandgap $(\mathbf{e V})$ & Dielectric constant & Refractive index & Reflectivity \\
\hline $\mathrm{SnO}_{2}$ & 3.33 & 2.0 & 1.40 & 0.03 \\
\hline $\mathrm{B}_{\mathrm{Sn}}: \mathrm{SnO}_{2}$ & 2.73 & 2.5 & 1.55 & 0.05 \\
\hline $\mathrm{B}_{\mathrm{i}}: \mathrm{SnO}_{2}$ & 3.72 & 3.8 & 1.95 & 0.1 \\
\hline $\mathrm{In}_{\mathrm{Sn}}: \mathrm{SnO}_{2}$ & 3.25 & 3 & 1.60 & 0.06 \\
\hline $\mathrm{In}_{\mathrm{i}}: \mathrm{SnO}_{2}$ & 3.48 & 3.7 & 1.85 & 0.095 \\
\hline
\end{tabular}

Table 2. The calculated electronic and optical constants.

index, there is an increase in the lower photon energies and on the contrary, there is a significant decrease in the upper energies. This is due to the optical dispersion that these structures have.

To provide a further insight into the optical properties of all these structures, in Fig. 5 we calculated the reflectivity, the optical conductivity, and the absorption of the structures. Reflectivity (see Fig. 5a) is an important property of these materials as it signifies the amount of photons that are reflected from the material. We have shown that the values at zero photon energy are calculated at 3\%, 5\%, 10\%, 6\% and 9.5\% for $\mathrm{SnO}_{2}, \mathrm{~B}_{\mathrm{Sn}}: \mathrm{SnO}_{2}$, $\mathrm{B}_{\mathrm{i}}: \mathrm{SnO}_{2}, \mathrm{In}_{\mathrm{Sn}}: \mathrm{SnO}_{2}$ and $\mathrm{In}_{\mathrm{i}}: \mathrm{SnO}_{2}$ respectively. We predicted that $\mathrm{B}_{\mathrm{i}}: \mathrm{SnO}_{2}$ has the highest reflectivity in the nearinfrared and visible regions compared to all the other cases. Importantly, all the examined cases encounter less than $15 \%$ reflectivity in the infrared and visible region; thus they can be used as antireflective coatings when the dopant concentration is at $2 \%^{41}$. In Table 2 we have gathered all the calculated electrical and optical constants for reference.

In Fig. 5b we present the optical conductivity of all the calculated structures. If a photon has higher energy than the optical bandgap then a transition occurs and an electron-hole pair (exciton) is generated. The mobility of these excitons represent the optical conductivity, which is an important parameter that is used to design optical detectors ${ }^{42}$. Due to the electronic charge neutrality, these excitons do not contribute to the electrical conductivity ${ }^{43}$. The highest excitonic features are calculated at $13.2 \mathrm{eV}$ for undoped, $\mathrm{B}_{\mathrm{Sn}}: \mathrm{SnO}_{2}$ and $\mathrm{In}_{\mathrm{Sn}}: \mathrm{SnO}_{2}$ cases while for the interstitial dopants they are shifted to $9 \mathrm{eV}$ and $10 \mathrm{eV}$ for the $\mathrm{B}$ and In, respectively. The optical conductivity also describes the losses for a wave with the same frequency. From the graph, we can conclude that $\mathrm{SnO}_{2}$ and $\mathrm{In}_{\mathrm{i}}: \mathrm{SnO}_{2}$ have lower losses than the other cases. Lastly, in Fig. $5 \mathrm{c}$, d we present the absorption co-efficiency. The absorption for the undoped case starts at $380 \mathrm{~nm}$, which is underestimated compared to the experimental value, which is approximately $400 \mathrm{~nm}^{44}$. It is seen that $\mathrm{In}_{\mathrm{i}}$ and $\mathrm{B}_{\mathrm{i}}$ have the highest absorption to the visible region.

From the above, we can conclude that $\mathrm{B}_{\mathrm{i}}$ and $\mathrm{In}_{\mathrm{i}}$ doped $\mathrm{SnO}_{2}$ exhibits interesting characteristics, constituting the material applicable to photovoltaic and photocatalytic devices.

Surface (110) of rutile $\mathrm{SnO}_{2}$. As Zhi et al. ${ }^{9}$ indicated to further understand the effect of $\mathrm{B}_{\mathrm{i}}$, the surface of $\mathrm{SnO}_{2}$ needs to be examined. For applicable photocatalytic materials with a visible light response and high charge mobility, such as $\mathrm{SnO}_{2}$, attention to the high-energy surfaces should be paid when the structure is doped with atoms or molecules. The studies of $\mathrm{SnO}_{2}$ surface are significantly less than the studies of the bulk and they primarily focus on sensor applications ${ }^{45}$. In this section, the change to the electronic properties of $B$ and In doped $\mathrm{SnO}_{2}$ will be investigated. Here we used a slab model with a vacuum of 12 A thickness across the (110) plane. We chose this particular surface because it is the most intense crystallization plane ${ }^{9,45}$ of rutile $\mathrm{SnO}_{2}$ and it has never been studied before for B or In doping. Furthermore, as the (110) plane is one of the most highly energetic surfaces, it will play the role of the active site in photocatalytic reactions ${ }^{46}$. Indium doping improves the electrical characteristics in $\mathrm{SnO}_{2}$ nanowire structures. Specifically, the field enhancement factor is higher compared to other dopants ${ }^{47-49}$. In these applications, the surface of the nanowire plays a significant role and it is responsible for the fluctuations in the field emission properties ${ }^{47}$.

Here we predicted the interstitial position of both B and In by examining many different configurations and keeping the lowest energy system. To accurate predict the electronic characteristics, we used the hybrid functional PBE0.

In Fig. 6a,b we present the $\mathrm{B}_{\mathrm{i}}: \mathrm{SnO}_{2}$ and $\mathrm{In}_{\mathrm{i}}: \mathrm{SnO}_{2}$ doping cases while in Fig. $6 \mathrm{c}$ the supercell for the (110) plane is presented for reference. The boron interstitial displaces the tin atom and occupies a tin site to minimize its energy (Fig. 6a). We predicted that interstitial boron sites at $2.182 \AA$ from the nearest oxygen atom and $3.10 \AA$ from the nearest tin atom. On the other hand, we calculated that In atom sites at a distance of $2.183 \AA$ from the nearest oxygen atom and $3.32 \AA$ from the nearest tin atom. Looking at the DOS and the pDOS of Fig. $7 \mathrm{a}$, it is seen that boron interstitial produces a small band peak at $1 \mathrm{eV}$.

Interestingly in this high-energy plane the bandgap is not decreased compared to the undoped case (see Fig. 7c). We believe that this result combined with the bulk $\mathrm{B}_{\mathrm{i}}: \mathrm{SnO}_{2}$ explains the experimental phenomenon that Zhi et al. ${ }^{9}$ discussed in their paper about $\mathrm{B}_{\mathrm{i}}$. Looking at the $\mathrm{In}_{\mathrm{i}}: \mathrm{SnO}_{2}$ (refer to Fig. $7 \mathrm{~b}$ ) we can see that the (110) surface bandgap decreases to $2 \mathrm{eV}$. Indium doping of the surface $\mathrm{SnO}_{2}$ improves the sensitivity of tin oxide based gas sensors as it significantly affects the crystallization of the samples ${ }^{50}$. The gap of the undoped (110) $\mathrm{SnO}_{2}$ is shown in Fig. $7 \mathrm{c}$ at $2.5 \mathrm{eV}$, a good agreement with previous DFT studies ${ }^{51}$. All the above results indicate that In doping in the $\mathrm{SnO}_{2}$ shows good characteristics that should be examined in photocatalytic applications. 

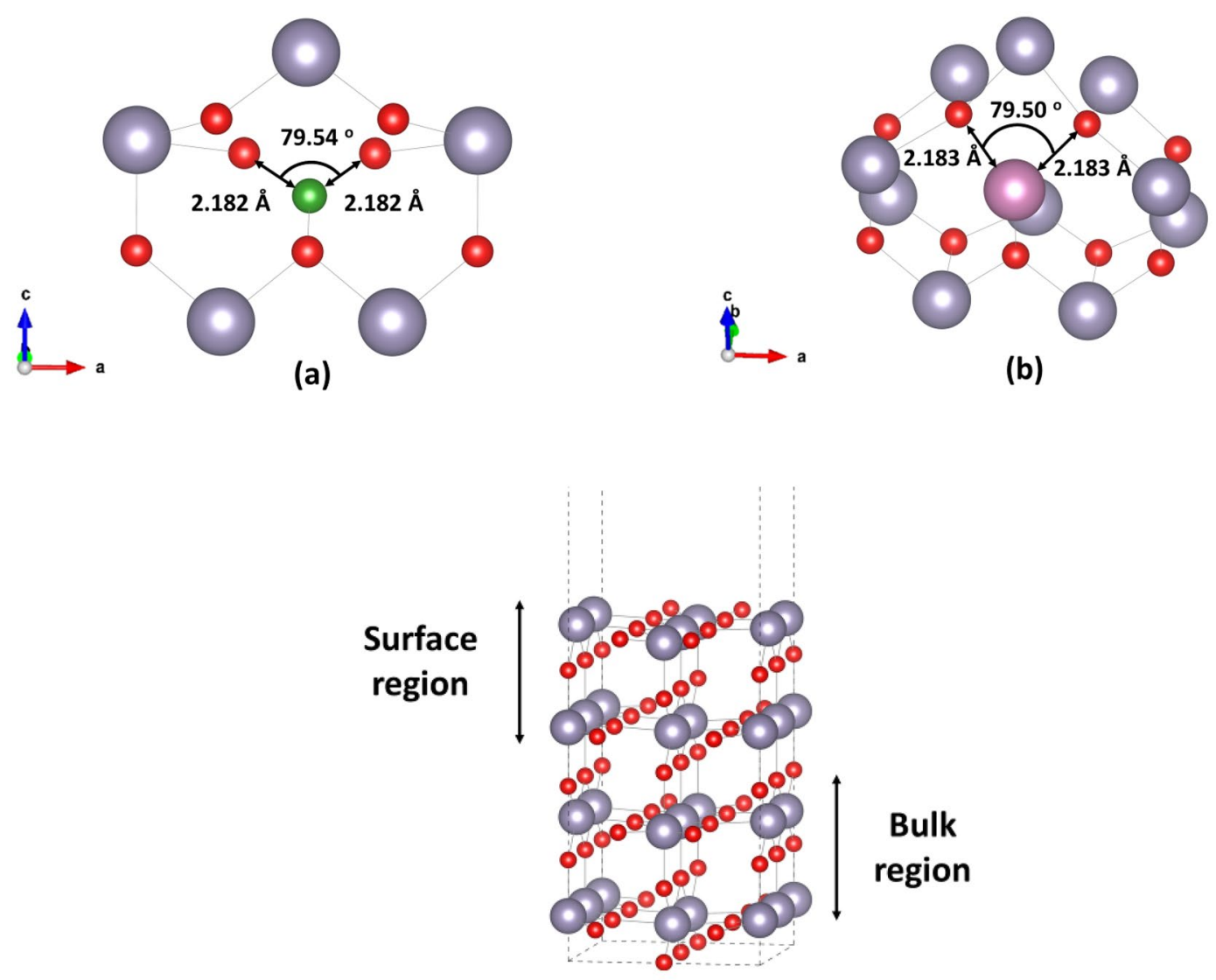

(c)

Figure 6. The structures of (a) boron doped (110) $\mathrm{SnO}_{2}$ surface, (b) indium doped (110) $\mathrm{SnO}_{2}$ surface and (c) the (110) surface supercell.
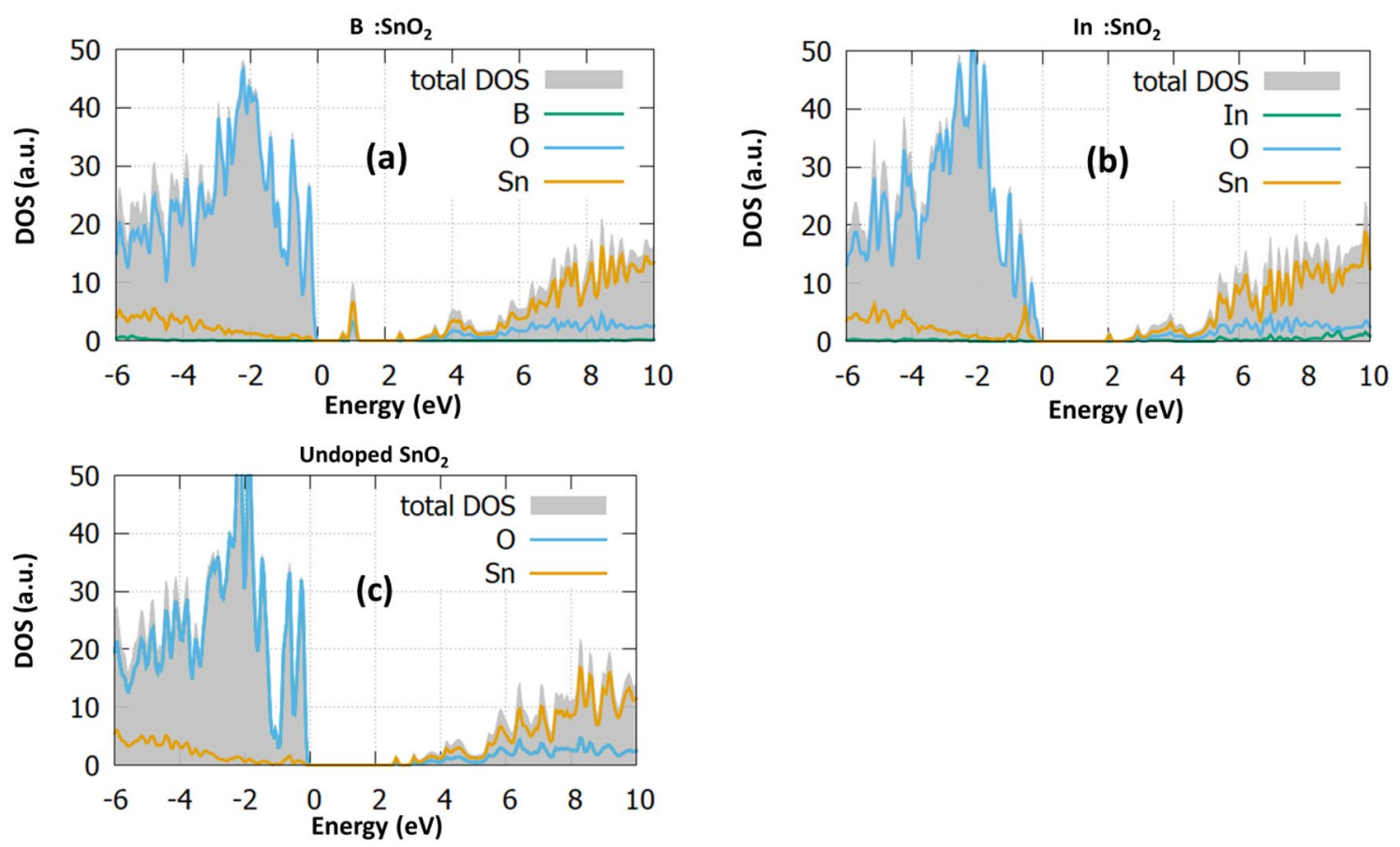

Figure 7. The total density of states (DOS) and the projected density of states (PDOS) of (a) B (b) In and (c) undoped (110) $\mathrm{SnO}_{2}$ surface. 


\section{Conclusions}

In the present study, we applied DFT calculations to investigate the electronic and optical properties of boron and indium doped bulk and surface $\mathrm{SnO}_{2}$. In particular, we performed DOS calculations and we found that the bulk structures have a bandgap increase for interstitial boron and indium doping. Conversely, for the substitutional cases, the bandgap is notable decreased. Mid-gap states are formed in all the examined cases. Although these states might be detrimental for photovoltaics as they may act as trap states for photogenerated carriers, they can be useful for photocatalytic applications based on $\mathrm{B}: \mathrm{SnO}_{2}$ and $\mathrm{In}: \mathrm{SnO}_{2}$. The present study explains some of the phenomena that have been experimentally observed and paves the way for applications of boron and indium structures to more applications such as supercapacitors or sensors. Regarding the indium defect, we predicted that at low concentrations highly wanted states are formed near the valence band, which can be beneficial for energy harvesting devices. Furthermore, its optical characteristics and the bandgap reduction at the surface make it a worthy candidate for photocatalysis.

\section{Methodology}

We employed the Cambridge Serial Total Energy Package (CASTEP) ${ }^{52,53}$. For our calculations we used the hybrid functional PBE0 in order to consider the effect of the localized electrons and the bandgap underestimation that is generally encountered in GGA and LDA ${ }^{54}$. The cutoff energy was chosen at $800 \mathrm{eV}$ after performing the convergence test and for our calculations we used a 48 atom supercell $(2 \times 2 \times 2$ unit cells $)$ with $2 \times 2 \times 3 \mathrm{k}$-points for the sampling of the Brillouin zone during the geometry optimization ${ }^{54}$. To predict the interstitial positions in all cases, we used geometry optimization and we examined all possible configurations. Finally, we kept the position, which provides the lowest energy system. For the simulation of the surface we used a slab model with a vacuum of approximately $12 \AA$ vertical to the (110) direction. In our system, the top 2 layers represent the surface while the bottom 2 are fixed and mimic the bulk region. For the DOS calculations we used a k-point mesh of $5 \times 5 \times 5$ for the bulk modelling while for the surface we applied a $3 \times 3 \times 1$ set. Finally, the convergence criteria for our simulations were chosen at $2.0 \cdot 10^{-5} \mathrm{eV} /$ atom for the SCF tolerance, $0.05 \mathrm{eV} / \AA$ for the force tolerance and $0.001 \AA$ for the Max displacement tolerance.

Received: 4 April 2021; Accepted: 3 June 2021

Published online: 22 June 2021

\section{References}

1. Searle, A. B. The Glazer's Book (The Technical Press, 1935).

2. Holleman, A. F. \& Wiberg, E. Inorganic Chemistry (Academic Press, 2001).

3. Greenwood, N. N. \& Earnshaw, A. Chemistry of the Elements (Elsevier, 2012).

4. Tountas, M. et al. Water-soluble lacunary polyoxometalates with excellent electron mobilities and hole blocking capabilities for high efficiency fluorescent and phosphorescent organic light emitting diodes. Adv. Funct. Mater. 26(16), 2655-2665 (2016).

5. Wang, C. et al. Ultrathin $\mathrm{SnO}_{2}$ nanosheets: Oriented attachment mechanism, nonstoichiometric defects, and enhanced lithium-ion battery performances. J. Phys. Chem. C 116(6), 4000-4011 (2012).

6. Rakhshani, A. E., Makdisi, Y. \& Ramazaniyan, H. A. Electronic and optical properties of fluorine-doped tin oxide films. J. Appl. Phys. 83(2), 1049-1057 (1998).

7. Filippatos, P. P., Kelaidis, N., Vasilopoulou, M., Davazoglou, D. \& Chroneos, A. Defect processes in halogen doped SnO 2 . Appl. Sci. 11(2), 551 (2021).

8. Minami, T. Transparent conducting oxide semiconductors for transparent electrodes. Semicond. Sci. Technol. 20, S35-S44 (2005).

9. Zhi, J., Zhou, M., Zhang, Z., Reiser, O. \& Huang, F. Interstitial boron-doped mesoporous semiconductor oxides for ultratransparent energy storage. Nat. Commun. 12(1), 1-12 (2021).

10. Kong, X. B., Li, F., Qi, Z. N., Qi, L. \& Yao, M. M. Boron-doped tin dioxide films for environmental applications. Surf. Rev. Lett. 24(05), 1750059 (2017).

11. Zhao, W., Ma, W. H., Chen, C. C., Zhao, J. C. \& Shuai, Z. G. Efficient degradation of toxic organic pollutants with $\mathrm{Ni}_{2} \mathrm{O} 3 / \mathrm{TiO}_{2-\mathrm{x}} \mathrm{B}_{\mathrm{x}}$ under visible irradiation. J. Am. Chem. Soc. 126, 4782 (2004).

12. Bagwasi, S., Tian, B., Zhang, J. \& Nasir, M. Synthesis, characterization and application of bismuth and boron co-doped TiO ${ }_{2}$ : A visible light active photocatalyst. Chem. Eng. J. 217, 108 (2013).

13. Tran, Q. P., Fang, J. S. \& Chin, T. S. Optical properties and boron doping-induced conduction-type change in $\mathrm{SnO}_{2}$ thin films. J. Electron. Mater 45(1), 349-356 (2016).

14. Aouaj, M. A., Diaz, R., Belayachi, A., Rueda, F. \& Lefdil, M. A. Comparative study of ITO and FTO thin films grown by spray pyrolysis. Mater. Res. Bull. 44, 1458 (2009).

15. Kulkarni, A. K., Lim, T., Khan, M. \& Schulz, K. H. Electrical, optical, and structural properties of indium-tin-oxide thin films deposited on polyethylene terephthalate substrates by RF sputtering. J. Vac. Sci. Technol. A 16, 1636 (1998).

16. Peng-Fei, L. et al. Electronic structure and optical properties of antimony-doped $\mathrm{SnO}_{2}$ from first-principle study. Commun. Theor. Phys. 57, 145 (2012).

17. Canestraro, C. D., Roman, L. S. \& Persson, C. Polarization dependence of the optical response in $\mathrm{SnO}_{2}$ and the effects from heavily F doping. Thin Solid Films 517(23), 6301-6304 (2009).

18. Rivera, R., Marcillo, F., Chamba, W., Puchaicela, P. \& Stashans, A. $\mathrm{SnO}_{2}$ physical and chemical properties due to the impurity doping. Lect. Notes Eng. Comp. 2, 814-818 (2013).

19. Velikokhatnyi, O. I. \& Kumta, P. N. Ab-initio study of fluorine-doped tin dioxide: A prospective catalyst support for water electrolysis. Phys. B 406(3), 471-477 (2011).

20. Golovanov, V., Golovanova, V., Kuisma, M. \& Rantala, T. T. Electron spin resonance parameters of cation vacancies in tin dioxide doped with fluorine and hydrogen. J. Appl. Phys. 114(14), 143907 (2013).

21. Oshima, M. \& Yoshino, K. Structural and electronic structure of $\mathrm{SnO}_{2}$ by the first-principle study. Trans Tech Stäfa 725, 265-268 (2012).

22. Govaerts, K., Partoens, B. \& Lamoen, D. Extended homologous series of Sn-O layered systems: A first-principles study. Solid State Commun. 243, 36-43 (2016).

23. Mallick, H. K., Zhang, Y., Pradhan, J., Sahoo, M. P. K. \& Pattanaik, A. K. Influence of particle size and defects on the optical, magnetic and electronic properties of $\mathrm{Al}$ doped $\mathrm{SnO}_{2}$ nanoparticles. J. Alloys Compd. 854, 156067 (2021). 
24. Duan, Y. et al. Effects of Ga doping and hollow structure on the band-structures and photovoltaic properties of $\mathrm{SnO}_{2}$ photoanode dye-sensitized solar cells. RSC Adv. 5(114), 93765-93772 (2015).

25. Perdew, J. P. \& Levy, M. Physical content of the exact Kohn-Sham orbital energies: Band gaps and derivative discontinuities. Phys. Rev. Lett. 51(20), 1884 (1983).

26. Zervos, M. et al. Epitaxial highly ordered $\mathrm{Sb}: \mathrm{SnO}_{2}$ nanowires grown by the vapor liquid solid mechanism on $\mathrm{m}-, \mathrm{r}-$ and a- $\mathrm{Al}_{2} \mathrm{O}_{3}$. Nanoscale Adv. 1, 1980-1990 (2019).

27. Zhang, B., Tian, Y., Zhang, J. X. \& Cai, W. The structural and electrical studies on the Boron-doped $\mathrm{SnO}_{2}$ films deposited by spray pyrolysis. Vacuum 85(11), 986-989 (2011).

28. Lekshmy, S. S. \& Joy, K. Structural and optoelectronic properties of indium doped $\mathrm{SnO}_{2}$ thin films deposited by sol gel technique. J. Mater. Sci. Mater. Electron. 25(4), 1664-1672 (2014).

29. Yu, J. et al. Structural and electronic properties of $\mathrm{SnO}_{2}$ doped with non-metal elements. J Nanotechnol. 11(1), 1321-1328 (2020).

30. Filippatos, P. P. et al. Defect processes in $\mathrm{F}$ and $\mathrm{Cl}$ doped anatase $\mathrm{TiO}_{2}$. Sci. Rep. 9, 19970 (2019).

31. Filippatos, P. P. et al. Preparation of hydrogen, fluorine and chlorine doped and co-doped titanium dioxide photocatalysts: A theoretical and experimental approach. Sci. Rep. 11, 5700 (2021).

32. Butcher, K., Hirshy, H., Perks, R. M., Wintrebert, M. \& Chen, P. P. T. Stoichiometry effects and the Moss-Burstein effect for InN. Phys. Status Solidi A 203, 66-74 (2006).

33. Simon, P. \& Gogotsi, Y. Materials for electrochemical capacitors. Nat. Mater. 7, 845-854 (2008).

34. Kumar, M., Kumar, A. \& Abhyankar, A. C. $\mathrm{SnO}_{2}$ based sensors with improved sensitivity and response-recovery time. Ceram. Int 40(6), 8411-8418 (2014).

35. Manassidis, I., Goniakowski, J., Kantorovich, L. N. \& Gillan, M. J. The structure of the stoichiometric and reduced $\mathrm{SnO}_{2}(110)$ surface. Surf. Sci. 339(3), 258-271 (1995).

36. Abdulsattar, M. A., Batros, S. S. \& Addie, A. J. Indium doped $\mathrm{SnO}_{2}$ nanostructures preparation and properties supported by DFT study. Superlattices Microstruct. 100, 342-349 (2016).

37. Tingting, S., Fuchun, Z. \& Weihu, Z. Density functional theory study on the electronic structure and optical properties of $\mathrm{SnO}_{2}$. Rare Metal Mater. Eng. 44(10), 2409-2414 (2015).

38. Khan, A. F., Mehmood, M., Aslam, M. \& Ashraf, M. Characteristics of electron beam evaporated nanocrystalline $\mathrm{SnO}_{2}$ thin films annealed in air. Appl. Surf. Sci. 256(7), 2252-2258 (2010).

39. Singh, A., Chatterjee, R., Mishra, S. K., Krishna, P. S. R. \& Chaplot, S. L. Origin of large dielectric constant in La modified $\mathrm{BiFeO}_{3}-$ $\mathrm{PbTiO}_{3}$ multiferroic. J. Appl. Phys. 111(1), 014113 (2012).

40. Afify, H. H., Momtaz, R. S., Badawy, W. A. \& Nasser, S. A. Some physical properties of fluorine-doped $\mathrm{SnO}_{2}$ films prepared by spray pyrolysis. J. Mater. Sci. Mater. Electron. 2(1), 40-45 (1991).

41. Khoshman, J. M. \& Kordesch, M. E. Optical properties of a-HfO ${ }_{2}$ thin films. Surf. Coat. Technol. 201(6), 3530-3535 (2006).

42. Akinlami, J. O. \& Olateju, I. O. Reflection coefficient and optical conductivity of gallium nitride GaN. Quant. Electron. Optoelectron. 15(3), 281-284 (2012).

43. Dash, L. K., Vast, N., Baranek, P., Reining, M. C. \& Cheynet, L. Electronic structure and electron energy-loss spectroscopy of $\mathrm{ZrO}_{2}$ zirconia. Phys. Rev. B 70(24), 245116 (2004).

44. Doyan, A., Susilawati, \& Imawanti, Y. D. Synthesis and characterization of $\mathrm{SnO}_{2}$ THIN layer with a doping aluminum is deposited on quartz substrates. AIP AIP Conf. Proc. 1801(1), 020005 (2017).

45. Wang, X., Qin, H., Chen, Y. \& Hu, J. Sensing mechanism of $\mathrm{SnO}_{2}(110)$ surface to CO: Density functional theory calculations. J. Chem. Phys. C 118(49), 28548-28561 (2014).

46. Talebian, N. \& Jafarinezhad, F. Morphology-controlled synthesis of $\mathrm{SnO}_{2}$ nanostructures using hydrothermal method and their photocatalytic applications. Ceram. Int. 39(7), 8311-8317 (2013).

47. Bhise, A. B. et al. A single In-doped $\mathrm{SnO}_{2}$ submicrometre sized wire as a field emitter. J. Phys. D: Appl. Phys. 40, 3644-3648 (2007).

48. Bhise, A. B. et al. Sb-doped $\mathrm{SnO}_{2}$ wire: Highly stable field emitter. J. Cryst. Growth 307, 87-91 (2007).

49. Bhise, A. B. et al. Field emission investigations of $\mathrm{RuO}_{2}$-doped $\mathrm{SnO}_{2}$ wires. Appl. Surf. Sci. 253, 9159-9163 (2007).

50. Cui, S. et al. Indium-doped $\mathrm{SnO}_{2}$ nanoparticle-graphene nanohybrids: Simple one-pot synthesis and their selective detection of $\mathrm{NO}_{2}$. J. Mater. Chem. A 1(14), 4462-4467 (2013).

51. Li, M., Zhu, H., Wei, G., He, A. \& Liu, Y. DFT calculation and analysis of the gas sensing mechanism of methoxy propanol on Ag decorated SnO2 (110) surface. RSC Adv. 9(61), 35862-35871 (2019).

52. Segall, M. D. et al. First-principles simulation: Ideas, illustrations and the CASTEP code. J. Phys. Cond. Matter 14(11), 2717-2744 (2002).

53. Ceperley, D. M. \& Alder, B. J. Exchange-correlation potential and energy for density-functional calculation. Phys. Rev. Lett. 45, 567-581 (1980).

54. Paier, J. et al. Screened hybrid density functionals applied to solids. J. Chem. Phys. 124, 154709 (2006).

\section{Acknowledgements}

P.P.F., M.V., D.D. and A.C. are grateful for LRF ICON funding from the Lloyd's Register Foundation, a charitable foundation helping to protect life and property by supporting engineering-related education, public engagement and the application of research. N.K. acknowledges support from European Union's H2020 Programme under Grant Agreement No 824072-HARVESTORE.

\section{Author contributions}

P.P.F. and N.K. performed the calculations. P.P.F., N.K., M.V., D.D. and A.C. contributed to the interpretation of the results and the writing of the paper.

\section{Competing interests}

The authors declare no competing interests.

\section{Additional information}

Correspondence and requests for materials should be addressed to A.C.

Reprints and permissions information is available at www.nature.com/reprints.

Publisher's note Springer Nature remains neutral with regard to jurisdictional claims in published maps and institutional affiliations. 
(c) (i) Open Access This article is licensed under a Creative Commons Attribution 4.0 International cc) License, which permits use, sharing, adaptation, distribution and reproduction in any medium or format, as long as you give appropriate credit to the original author(s) and the source, provide a link to the Creative Commons licence, and indicate if changes were made. The images or other third party material in this article are included in the article's Creative Commons licence, unless indicated otherwise in a credit line to the material. If material is not included in the article's Creative Commons licence and your intended use is not permitted by statutory regulation or exceeds the permitted use, you will need to obtain permission directly from the copyright holder. To view a copy of this licence, visit http://creativecommons.org/licenses/by/4.0/.

(C) The Author(s) 2021 